\title{
Deep brain stimulation for the obsessive-compulsive and Tourette-like symptoms of Kleefstra syndrome
}

\author{
David J. Segar, BS, ${ }^{1}$ Yosef G. Chodakiewitz, BA, ${ }^{1}$ Radmehr Torabi, MD,, 2 and \\ G. Rees Cosgrove, MD, FRCS ${ }^{1,2}$
}

\begin{abstract}
${ }^{1}$ Alpert Medical School, Brown University; and 'Department of Neurosurgery, Rhode Island Hospital, Providence, Rhode Island
Deep brain stimulation (DBS) has been reported to have beneficial effects in severe, treatment-refractory cases of obsessive-compulsive disorder (OCD) and Tourette syndrome (TS). In this report, the authors present the first case in which DBS was used to treat the neuropsychiatric symptoms of Kleefstra syndrome, a rare genetic disorder characterized by childhood hypotonia, intellectual disability, distinctive facial features, and myriad psychiatric and behavioral disturbances. A 24-year-old female patient with childhood hypotonia, developmental delay, and diagnoses of autism spectrum disorder, OCD, and TS refractory to medical management underwent the placement of bilateral ventral capsule/ventral striatum (VC/VS) DBS leads, with clinical improvement. Medical providers and family observed gradual and progressive improvement in the patient's compulsive behaviors, coprolalia, speech, and social interaction. Symptoms recurred when both DBS electrodes failed because of lead fracture and dislodgement, although the clinical benefits were restored by lead replacement. The symptomatic and functional improvements observed in this case of VC/VS DBS for Kleefstra syndrome suggest a novel indication for DBS worthy of further investigation.
\end{abstract}

http://thejns.org/doi/abs/10.3171/2015.3.FOCUS1528

KEY WORDS Kleefstra syndrome; deep brain stimulation; obsessive-compulsive disorder; ventral striatum; EHMT1 protein; human

$\mathrm{K}$ LEEFSTRA syndrome is a rare genetic disorder that results from disruption of the euchromatic histone-lysine- $N$-methyltransferase 1 (EHMT1) gene as the result of either a chromosomal microdeletion or an EHMT1 mutation. ${ }^{25}$ Characterized by a phenotype of childhood hypotonia, intellectual disability, and distinctive facial features, this syndrome is often accompanied by myriad psychiatric and behavioral disorders. ${ }^{12,22,25}$

While deep brain stimulation (DBS) has been used as a treatment for a number of neuropsychiatric conditions including obsessive-compulsive disorder (OCD), depression, and Tourette syndrome (TS), there is no literature on using DBS for the neuropsychiatric symptoms of a purely genetic disorder. In 1999, Nuttin and colleagues ${ }^{18}$ were the first to describe the use of DBS for OCD, and their encouraging initial experience was confirmed with additional studies, ${ }^{8,9,19}$ resulting in FDA approval of DBS for the treatment of OCD. Multiple reports also suggest beneficial effects in severe, treatment-refractory cases of TS.,23 The present report describes the first case in which DBS was used to treat the obsessive-compulsive and TS-like features of Kleefstra syndrome, a rare genetic disorder with no currently effective treatments.

\section{Case Report}

History and Examination

A 24-year-old female presented with childhood hypotonia, developmental delay, and subsequent regression. In adolescence, she had become less communicative, had demonstrated decreases in her reading ability, and had been unable to manage activities of daily living. On presentation, she had a baseline global assessment of functioning (GAF) score of 20, indicative of severe impairment.

Family members reported that she spent a significant portion of her time standing staring at traffic or repetitively turning the water in the sink on and off, becoming distressed if interrupted. She demonstrated multiple stereotypic movements, biting her hands and picking at her skin to the point of bleeding. Communication was limited to

ABBREVIATIONS AC = anterior commissure; DBS = deep brain stimulation; GAF = global assessment of functioning; OCD = obsessive-compulsive disorder; $\mathrm{PC}=$ posterior commissure; TS = Tourette syndrome; VC/VS = ventral capsule/ventral striatum; YBOCS = Yale-Brown Obsessive Compulsive Scale.

SUBMITTED February 1, 2015. ACCEPTED March 9, 2015.

INCLUDE WHEN CITING DOI: 10.3171/2015.3.FOCUS1528.

DISCLOSURE The authors report no conflict of interest concerning the materials or methods used in this study or the findings specified in this paper. 
single words or short phrases. This constellation of symptoms led to diagnoses of pervasive developmental disorder and OCD. She was additionally noted to have severe coprolalia and vocal tics consistent with TS. Over time, her neuropsychiatric symptoms continued to worsen and became incapacitating despite multiple attempts at medical and behavioral therapy.

\section{Operation}

Following discussion among the patient's multidisciplinary medical team and family, the patient underwent bilateral placement of ventral capsule/ventral striatum (VC/VS) DBS leads (Medtronic 3389) to treat her disabling OCD and TS; an outside neurosurgeon performed the initial electrode placement. Target coordinates were chosen as previously described for the VC/VS region used in OCD trials. ${ }^{8,9,19}$ Correct implantation was confirmed using postoperative $\mathrm{CT}$ studies merged with preoperative MRI. Positioning of the distal electrode contacts was determined from postimplantation imaging, using the posterior border of the anterior commissure (AC), posterior commissure (PC), and AC-PC line as reference. Bilaterally, distal contacts were determined to be $7 \mathrm{~mm}$ lateral to the midline and $3 \mathrm{~mm}$ ventral to the AC-PC line. Anteroposterior positioning of the left electrode was $1 \mathrm{~mm}$ anterior to the $\mathrm{AC}$, while the right electrode was located at the level of the AC. These parameters are within the range of effective $\mathrm{VC} / \mathrm{VS}$ electrode placements determined in previous cases of VC/VS DBS for OCD. ${ }^{8}$

\section{Postoperative Course}

Stimulation parameters were consistent with those previously described at this target. ${ }^{9119}$ Stimulation was tested at a range of 2-8 V, and initial therapy was started at $6 \mathrm{~V}$, with a frequency of $130 \mathrm{~Hz}$ and a pulse width of $90 \mu \mathrm{sec}$. The patient's clinical course was monitored while on and off stimulation. Objective assessment with the Yale-Brown Obsessive Compulsive Scale (YBOCS) was attempted to precisely chart OCD severity and response to treatments; however, the scale could not be applied consistently given the patient's underlying intellectual disability. Instead, the involved medical providers and her family subjectively determined her psychiatric state. Additionally, GAF scores were determined at various stages of treatment.

After implantation, the patient demonstrated gradual and progressive improvement in her obsessive-compulsive behaviors, coprolalia, speech, and social interaction over 3-9 months. Her GAF scores improved steadily during this time, jumping to 30-40 shortly after implantation and increasing to 50-60 after 9 months.

She then began to gradually regress clinically over several months with noted worsening of symptoms. Imaging revealed that one DBS lead had fractured and the other had withdrawn from the target (Fig. 1). By this time, her GAF score had returned to a baseline of 20 . Both leads were subsequently explanted. Because medical providers, teachers, caregivers, and family corroborated that DBS had dramatically improved the patient's function, the leads were replaced in their original locations in the $\mathrm{VC} /$ VS. Implantation was again confirmed with postoperative CT studies merged with preoperative MRI (Fig. 2).
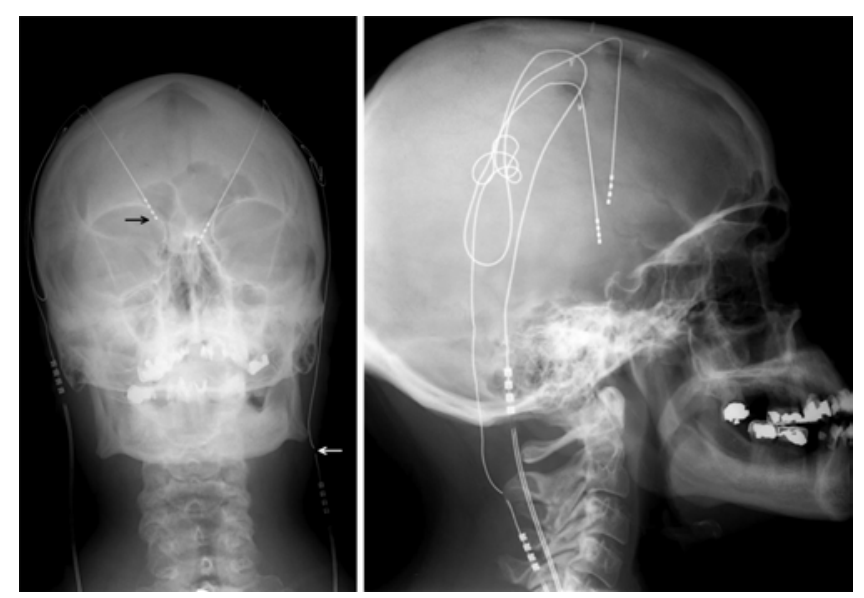

FIG. 1. Plain skull radiographs demonstrating lead fracture of the left DBS electrode (white arrow) and withdrawal and displacement of the right DBS lead from the target area (black arrow).

Following lead replacement, the patient once again began to experience substantial improvement, with a similar increase in GAF scores to between 50 and 60 . She became more communicative and interactive at school, an improvement that was noted by clinicians, family members, teachers, other parents, and students. She became significantly more interactive and motivated during daily activities and even participated in Special Olympics bowling. Care providers also noted a significant reduction in her anxiety, compulsive behaviors, coprolalia, and vocal tics. Her hyperphagic tendencies also decreased, contributing to a nearly $20-\mathrm{kg}$ weight loss.

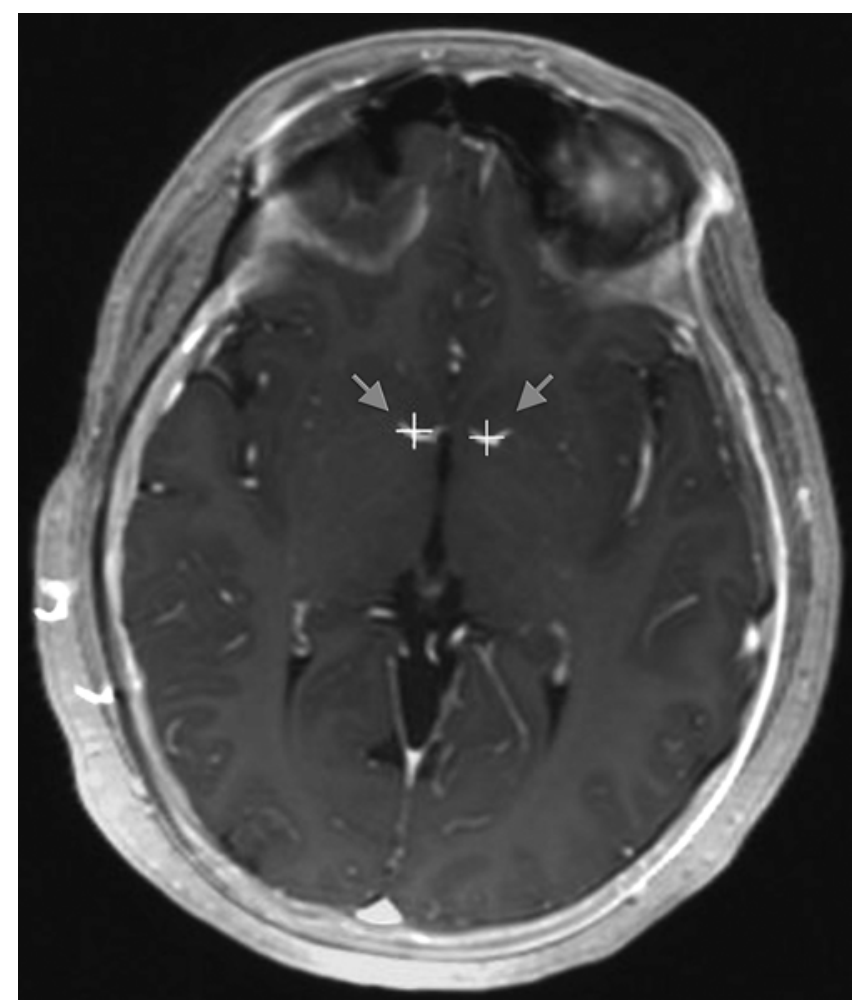

FIG. 2. Preoperative MR image fused with postoperative CT scan demonstrating effective VC/VS electrode replacement. 
Approximately 2 years following lead replacement, Kleefstra syndrome was diagnosed after the detection of a 9q34.3 deletion on microarray. Despite the observation that some patients with Kleefstra syndrome demonstrate a regressive course with worsening motor and neurobehavioral symptoms over time, ${ }^{24}$ our patient continued to function substantially above her baseline level more than 3 years after replacement of the DBS leads. A considerable reduction in OCD behaviors has allowed for substantial qualitative benefits to the patient and her family. Occasional subtle worsening of symptoms over the more than 3 years since lead replacement has largely been reversed or improved by adjusting stimulation parameters. At the last follow-up, the stimulus amplitude was set to $8 \mathrm{~V}$, with a frequency of $130 \mathrm{~Hz}$ and a pulse width of $90 \mu \mathrm{sec}$. Because of the frequent battery changes necessary at these settings, both pulse generators were subsequently replaced with versions that could be externally recharged.

At the patient's last follow-up, 3 years after replacement of her DBS leads, she continued to demonstrate sustained and substantial improvement in her OCD symptoms, TSlike tics, coprolalia, and social behavior and sustained improvement in her GAF.

\section{Discussion}

Of the 85 cases of Kleefstra syndrome reported since the EHMT1 gene was discovered in the 1990s, a significant majority have been associated with the 9q34.3 microdeletion. ${ }^{25}$ Among patients with this microdeletion, 54\% have behavioral and psychiatric problems, 58\% demonstrate abnormalities on brain imaging, $36 \%$ have a concurrent diagnosis of epilepsy, and $20 \%$ are defined as obese. ${ }^{25}$ The syndrome affects multiple organ systems, and the phenotype commonly includes brachycephaly, prognathism, macroglossia, tented upper lip, everted lower lip, and conotruncal defects. ${ }^{12,22,25}$

The behavioral and neuropsychiatric symptoms alleviated in our patient are common in Kleefstra syndrome but are frequently accompanied by other traits. Patients may demonstrate, with variable penetrance, autistic features, concentration and attention deficits, sleep disorders, anxiety, obsessive-compulsive symptoms, stereotypic movements and motor disorders, severe speech delay, lack of social interaction, and posturing. ${ }^{12,22,24}$ There is some evidence of a neurodegenerative, progressive apathy syndrome that becomes more evident in adolescence and adulthood. However, minimal literature documents the prevalence of such behavioral phenotypes. ${ }^{24}$

While the current literature does report obsessivecompulsive symptoms, bursts of anger, and disorders of attention as common, ${ }^{12}$ a phenotypic prevalence can only be estimated. Similarly, a review of the literature failed to demonstrate specific mention of TS in patients with Kleefstra syndrome; however, TS is a common comorbidity of both $\mathrm{OCD}^{11}$ and autism spectrum disorders, ${ }^{4}$ and the two are associated with Kleefstra syndrome. ${ }^{22,25}$

Multiple possible DBS targets have been examined for the treatment of OCD, including the anterior limb of the internal capsule, nucleus accumbens, VC/VS, subthalamic nucleus, and inferior thalamic peduncle. ${ }^{13}$ A systematic re- view by Kohl et al. in 2014 demonstrated approximately equivalent reductions in OCD severity across the first 4 targets, while the inferior thalamic peduncle had higher success rates but a much smaller sample size. ${ }^{13}$ While unequivocally determining a superior target may be difficult, the largest of the reviewed studies, by Greenberg et al., noted that results improved with slight posterior adjustments to VC/VS targeting over the course of the study, leading to improved outcomes at lower average stimulation requirements. ${ }^{8}$ Electrode placement varied from an average of more than $4 \mathrm{~mm}$ anterior to the $\mathrm{AC}$ in the first cohort to an average centered around $0 \mathrm{~mm}$ anterior to the $\mathrm{AC}$ in the final group of patients, in whom targeting was considered to be the most effective. Targeting in this final group was consistent with the location of the DBS electrodes in our patient. Moreover, the stimulation parameters tested and used ranged from 2 to $8 \mathrm{~V},{ }^{8}$ consistent with the parameters used in our patient.

The effects of DBS on TS have only been described in a small number of patients. Multiple targets have been successful on a case-by-case basis, with the largest number of patients treated with bilateral implantation in the centromedian-parafascicular complex and ventralis oralis of the thalamus. ${ }^{21}$ Other DBS targets, including the internal segment of the globus pallidus, ,5,, 16 anterior limb of the internal capsule, ${ }^{7,14}$ and nucleus accumbens, ${ }^{14,17}$ have demonstrated a successful reduction in tic severity and comorbid obsessive-compulsive symptoms, a common clinical finding in patients with TS. ${ }^{15}$ A reported case of TS with severe comorbid OCD and self-mutilation responded dramatically to DBS in the nucleus accumbens. ${ }^{17}$ These latter targets are most consistent with the target used in our patient. In reviewing the stimulation parameters in cases of nucleus accumbens and anterior internal capsule stimulation, we found initial voltages ranging from 4 to $7 \mathrm{~V}$.,14,17

The juxtaposed anatomy of the anterior limb of the internal capsule, nucleus accumbens, and VC/VS allows targeting of this area to modulate connections considered relevant to both OCD and TS. Successful intervention at these locations is consistent with the disruption of orbitofrontal-caudate-pallidal-thalamic circuitry, implicated as a source of possible hyperactivity contributing to OCD and TS symptoms. ${ }^{10}$

Studies of VC/VS DBS for severe OCD have consistently noted improvements in disease severity as determined by clinical assessment and the YBOCS. In a multisite study of 26 severe OCD cases (mean preimplantation YBOCS score of 34), patients demonstrated an average decrease to 20.9 in the YBOCS score at 36 months of active DBS, well below the study definition of severe OCD. ${ }^{8}$ At 1 month of treatment, $28 \%$ of patients met criteria for a full response rate of a $35 \%$ decrease in the YBOCS score. Notably, full response rates continued to rise over time, increasing to $61.5 \%$ by the last follow-up. An additional $11.5 \%$ of patients who did not meet the criteria for a full response improved at least $25 \%$.

This same study demonstrated marked improvement in global functioning, as measured by GAF scores. ${ }^{8}$ Twenty of the 21 patients in whom GAF scores were available had baseline scores below the cutoff of 40, consistent with major functional impairment. At the last follow-up, mean 
GAF scores had risen from a baseline of 34.8 to 59.0. These data are consistent with the improvements in global functioning noted in our case. Patients also demonstrated consistent global improvement on a 5-point clinical scale, with $65 \%$ noted to be "much better" and no patient found to be worse than baseline. ${ }^{8}$

In our case, subjective evidence, albeit from diverse sources-mother, doctors, teachers, friends, and neighbors-pointed to significant clinical improvement in anxiety, compulsive symptoms, vocal tics, and general functioning after successful DBS. These changes are consistent with improvements seen in other cases of DBS for OCD and TS. Our ability to assess our patient was limited by the inability to use validated rating scales, such as the YBOCS or tic severity scale, and such widely accepted measures would have allowed for more detailed monitoring of symptom evolution over time. Despite our inability to rely on such effective assessments, the perspicuous recurrence of symptoms with DBS lead failure and the restoration of benefit after reimplantation solidify the assertion that $\mathrm{VC} /$ VS DBS was in fact responsible for the substantial clinical improvement in this case. Given the failure of both the right and left leads prior to reimplantation, it is difficult to assess the effectiveness of unilateral DBS on symptom control in our patient; however, we must consider the possibility that significant symptom control was achieved with unilateral electrode function and that the lead malfunction was only clinically unmasked after failure of the second lead.

It should be noted that DBS is a procedure that requires meticulous attention to detail to avoid technical pitfalls. In this case, proper lead placement was verified after the initial operation by using postoperative CT studies merged with MRI. Months later, right-sided lead migration was noted, a relatively uncommon late complication. ${ }^{3,20}$ Moreover, the left lead was found to be fractured, a far more common complication probably caused by repetitive disruption of a cervically placed lead during movement. ${ }^{20}$ It is likely that both of these events resulted from inadequate anchoring of the connectors, which caused downward migration from their initial positions on the patient's skull. ${ }^{20}$

\section{Conclusions}

Kleefstra syndrome is a heterogeneous and multisystem genetic disorder that cannot be managed with a single intervention. Instead, the provision of optimal care requires the attention of multiple disciplines, and in our patient we present a new and promising treatment modality. The symptomatic and functional improvements observed in this first case of VC/VS DBS for Kleefstra syndrome suggest a novel indication for DBS worthy of further investigation. The presence of OCD and TS symptoms in a monogenetic disease such as Kleefstra syndrome offers an intriguing avenue for the study of both disease and treatment through the exploration of animal models. Initial studies in EHMTI knockout mice have demonstrated some behavioral abnormalities proposed to be consistent with the intellectual disability, autistic features, and anxiety seen in Kleefstra syndrome, ${ }^{1,2}$ and further study of these models will, we hope, provide additional insight. The decline in function following electrode malfunction and subsequent recovery following reimplantation implicates DBS as the likely causal factor for the patient's improvement. This correlation, together with the longevity of ongoing benefits to the patient, suggests a possible therapeutic role for DBS in patients with this rare genetic disorder.

\section{Acknowledgments}

We acknowledge the excellent clinical assessments and care provided by John Gaitanis, MD, Wael Asaad, MD, PhD, and Chanika Phornphutkul, MD.

\section{References}

1. Balemans MC, Huibers MM, Eikelenboom NW, Kuipers AJ, van Summeren RC, Pijpers MM, et al: Reduced exploration, increased anxiety, and altered social behavior: Autistic-like features of euchromatin histone methyltransferase 1 heterozygous knockout mice. Behav Brain Res 208:47-55, 2010

2. Balemans MCM, Kasri NN, Kopanitsa MV, Afinowi NO, Ramakers G, Peters TA, et al: Hippocampal dysfunction in the Euchromatin histone methyltransferase 1 heterozygous knockout mouse model for Kleefstra syndrome. Hum Mol Genet 22:852-866, 2013

3. Blomstedt P, Hariz MI: Hardware-related complications of deep brain stimulation: a ten year experience. Acta Neurochir (Wien) 147:1061-1064, 2005

4. Canitano R, Vivanti G: Tics and Tourette syndrome in autism spectrum disorders. Autism 11:19-28, 2007

5. Dehning S, Mehrkens JH, Müller N, Bötzel K: Therapyrefractory Tourette syndrome: beneficial outcome with globus pallidus internus deep brain stimulation. Mov Disord 23:1300-1302, 2008

6. Diederich NJ, Kalteis K, Stamenkovic M, Pieri V, Alesch F: Efficient internal pallidal stimulation in Gilles de la Tourette syndrome: a case report. Mov Disord 20:1496-1499, 2005

7. Flaherty AW, Williams ZM, Amirnovin R, Kasper E, Rauch SL, Cosgrove GR, et al: Deep brain stimulation of the anterior internal capsule for the treatment of Tourette syndrome: technical case report. Neurosurgery 57 (4 Suppl):E403, 2005

8. Greenberg BD, Gabriels LA, Malone DA Jr, Rezai AR, Friehs GM, Okun MS, et al: Deep brain stimulation of the ventral internal capsule/ventral striatum for obsessivecompulsive disorder: worldwide experience. Mol Psychiatry 15:64-79, 2010

9. Greenberg BD, Malone DA, Friehs GM, Rezai AR, Kubu CS, Malloy PF, et al: Three-year outcomes in deep brain stimulation for highly resistant obsessive-compulsive disorder. Neuropsychopharmacology 31:2384-2393, 2006

10. Greenberg BD, Rauch SL, Haber SN: Invasive circuitrybased neurotherapeutics: stereotactic ablation and deep brain stimulation for OCD. Neuropsychopharmacology 35:317336, 2010

11. Hallett M: Tourette syndrome: update. Brain Dev [epub ahead of print], 2015

12. Kleefstra T, Brunner HG, Amiel J, Oudakker AR, Nillesen WM, Magee A, et al: Loss-of-function mutations in euchromatin histone methyl transferase 1 (EHMT1) cause the 9q34 subtelomeric deletion syndrome. Am J Hum Genet 79:370377, 2006

13. Kohl S, Schönherr DM, Luigjes J, Denys D, Mueller UJ, Lenartz D, et al: Deep brain stimulation for treatment-refractory obsessive compulsive disorder: a systematic review. BMC Psychiatry 14:214, 2014

14. Kuhn J, Lenartz D, Mai JK, Huff W, Lee SH: Deep brain stimulation of the nucleus accumbens and the internal cap- 
sule in therapeutically refractory Tourette-syndrome. J Neurol 254:963-965, 2007

15. Leckman JF: Tourette's syndrome. Lancet 360:1577-1586, 2002

16. Martínez-Fernández R, Zrinzo L, Aviles-Olmos I, Hariz M, Martinez-Torres I, Joyce E, et al: Deep brain stimulation for Gilles de la Tourette syndrome: a case series targeting subregions of the globus pallidus internus. Mov Disord 26:19221930, 2011

17. Neuner I, Podoll K, Lenartz D, Sturm V, Schneider F: Deep brain stimulation in the nucleus accumbens for intractable Tourette's syndrome: follow-up report of 36 months. Biol Psychiatry 65:e5-e6, 2009

18. Nuttin B, Cosyns P, Demeulemeester H, Gybels J, Meyerson B: Electrical stimulation in anterior limbs of internal capsules in patients with obsessive-compulsive disorder. Lancet 354:1526, 1999

19. Nuttin BJ, Gabriëls LA, Cosyns PR, Meyerson BA, Andréewitch S, Sunaert SG, et al: Long-term electrical capsular stimulation in patients with obsessive-compulsive disorder. Neurosurgery 52:1263-1274, 2003

20. Oh MY, Abosch A, Kim SH, Lang AE, Lozano AM: Longterm hardware-related complications of deep brain stimulation. Neurosurgery 50:1268-1276, 2002

21. Servello D, Porta M, Sassi M, Brambilla A, Robertson MM: Deep brain stimulation in 18 patients with severe Gilles de la Tourette syndrome refractory to treatment: the surgery and stimulation. J Neurol Neurosurg Psychiatry 79:136-142, 2008

22. Stewart DR, Kleefstra T: The chromosome 9q subtelomere deletion syndrome. Am J Med Genet C Semin Med Genet 145C:383-392, 2007

23. Vandewalle V, van der Linden C, Groenewegen HJ, Cae- maert J: Stereotactic treatment of Gilles de la Tourette syndrome by high frequency stimulation of thalamus. Lancet 353:724, 1999

24. Verhoeven WMA, Egger JIM, Vermeulen K, van de Warrenburg BP, Kleefstra T: Kleefstra syndrome in three adult patients: further delineation of the behavioral and neurological phenotype shows aspects of a neurodegenerative course. Am J Med Genet A 155A:2409-2415, 2011

25. Willemsen MH, Vulto-van Silfhout AT, Nillesen WM, Wissink-Lindhout WM, van Bokhoven H, Philip N, et al: Update on Kleefstra syndrome. Mol Syndromol 2:202-212, 2012

\section{Author Contributions}

Conception and design: all authors. Acquisition of data: all authors. Analysis and interpretation of data: all authors. Drafting the article: all authors. Critically revising the article: all authors. Reviewed submitted version of manuscript: all authors. Approved the final version of the manuscript on behalf of all authors: Segar.

\section{Supplemental Information \\ Previous Presentation}

Portions of this work were presented in poster form at the Congress of the European Society for Stereotactic and Functional Neurosurgery held in Maastricht, The Netherlands, on September $17-20,2014$.

\section{Correspondence}

David J. Segar, Brown University, Box G-M212, Providence, RI 02912.email: david_segar@brown.edu. 